\title{
DT-13 inhibits cancer cell migration by regulating NMIIA indirectly in the tumor microenvironment
}

\author{
HONGZHI DU ${ }^{1}$, YUE HUANG ${ }^{1}$, XIAOYIN HOU ${ }^{1}$, XIAOWEN YU ${ }^{1}$, SENSEN LIN $^{2}$, XIAOHUI WEI $^{2}$, \\ RUIMING LI ${ }^{3}$, GHULAM JILANY KHAN ${ }^{1}$, SHENGTAO YUAN ${ }^{2}$ and LI SUN ${ }^{1}$ \\ ${ }^{1}$ Jiangsu Key Laboratory of Drug Screening, China Pharmaceutical University, Nanjing, Jiangsu 210009; \\ ${ }^{2}$ Jiangsu Center for Pharmacodynamics Research and Evaluation, China Pharmaceutical University, Nanjing, Jiangsu 210009; \\ ${ }^{3}$ Tasly Research Institute, Tianjin Tasly Holding Group Co., Ltd., Tianjin 300410, P.R. China
}

Received January 18, 2016; Accepted February 11, 2016

DOI: $10.3892 /$ or.2016.4890

\begin{abstract}
Tumor metastasis is one of the main causes of mortality among patients with malignant tumors. Previous studies concerning tumor metastasis have merely focused on the cancer cells in the tumor. However, an increasing number of studies show that the tumor microenvironment plays a vital role in the progression of cancer, particularly in tumor metastasis. Since fibroblasts and adipocytes are two of the most representative mesenchymal cells in the tumor microenvironment, we established a hypoxia-induced cancer-associated fibroblast (CAF) model and a chemically induced adipocyte model to reveal the effect of the microenvironment on cancer development. In these models, the conditioned medium from the tumor microenvironment was found to significantly promote the migration of human lung cancer cell line $95 \mathrm{D}$ and regulate the expression of non-muscle myosin IIA (NMIIA), which is consistent with results in the published literature. Then, we confirmed the hypothesis that the tumor microenvironment can regulate NMIIA in cancer cells and facilitate migration by using the nonmuscle myosin II inhibitor, blebbistatin. Thus, this is the first report that the tumor microenvironment can promote cancer cell migration by regulating the expression of NMIIA. Our present data also indicated that DT-13, the saponin monomer 13 of dwarf lilyturf tuber, inhibited cancer cell migration in the tumor microenvironment model. Further results showed that DT-13 exhibited anti-migratory effects by inhibiting the c-raf/ERK1/2 signaling pathway. Consequently, our research
\end{abstract}

Correspondence to: Professor Shengtao Yuan, Jiangsu Center for Pharmacodynamics Research and Evaluation, China Pharmaceutical University, 24 Tongjiaxiang, Nanjing, Jiangsu 210009, P.R. China E-mail: cpuyuanst@163.com

Professor Li Sun, Jiangsu Key Laboratory of Drug Screening, China Pharmaceutical University, 24 Tongjiaxiang, Nanjing, Jiangsu 210009, P.R. China

E-mail: cpusunli@126.com

Key words: hypoxia, tumor microenvironment, migration, DT-13, non-muscle myosin IIA confirmed that DT-13 significantly inhibited 95D cell migration in vitro, indicating the potential anti-metastatic effect of DT-13 on lung cancer and the scientific basis for drug development.

\section{Introduction}

China is facing a health crisis caused by cancer with nearly 2 million cancer-related deaths and more over 3 million cancer cases diagnosed annually (1). Unfortunately, tumor metastasis is one of the main causes of mortality among patients with malignant tumors (2). Previous research concerning tumor metastasis has focused on the adhesion and migratory ability of cancer cells themselves. However, an increasing number of studies show that the tumor microenvironment plays a vitally important role in the progression of cancer, especially in tumor metastasis (3). As a complex integrated system, the tumor microenvironment consists of fibroblasts, adipocytes, immune cells, inflammatory cells, endothelial cells and extracellular matrix (4). In the tumor microenvironment, the fibroblast is a specific cell type located in the stroma of cancer, namely cancer-associated fibroblast cells (CAFs). Accumulating evidence indicates that CAFs support the proliferation, migration and chemotherapy resistance of cancer cells by producing extracellular matrix, secreting cytokines, and activating signaling pathways in cancer cells $(5,6)$. The adipocyte, another mesenchymal cell, stores energy and also secretes various adipokines and cytokines to promote tumor development. During the interaction with the tumor microenvironment, adipocytes may dedifferentiate into pre-adipocytes or cancer-associated adipocytes (CAAs) which can stimulate the migration and invasion of cancer cells $(7,8)$. Obviously, fibroblasts and adipocytes as representative mesenchymal cells can be considered as targets for cancer therapy.

The cytoskeleton is essential for directional cell motility and migration. Actin cytoskeleton is the major player in cell motility and locomotion in most eukaryotic cells. Meanwhile, non-muscle myosin II (NMII) is a member of the myosin II subfamily, namely actin-based myosin motor protein, and NMIIA is a member of the NMII subclass (9). Previous evidence has demonstrated that NMIIA functions in cell adhesion, motility and migration, especially in cancer cell migration (10-14). Defects or low expression of NMIIA can 
promote cancer cell motility and migration via lamellipodia extension, decrease in focal adhesion, and upregulation of paxillin/p-paxillin (15-17). Thus, NMIIA may be a significant potential anti-metastatic target in cancer with high metastatic potential.

DT-13, the saponin monomer 13 of dwarf lilyturf tuber, is isolated from Ophiopogon japonicus (Thunb.) Ker-Gaul and is widely used in traditional Chinese medicine (TCM). DT-13 has been found to exhibit antitumor effects, especially anti-metastatic effects in vitro and in vivo (18-21). Our previous research showed that DT-13 could exhibit anti-metastatic effects directly by regulating NMIIA especially in lung cancer 95D cells (22). However, it is unknown whether DT-13 can inhibit cell migration by regulating NMIIA in cancer cells indirectly in the umor microenvironment. In the present study, we revealed the indirect metastatic inhibitory effect of DT-13 by regulating NMIIA in cancer cells through establishing a CAF-like myofibroblastic phenotype model by exposure to hypoxia, as in previous reports $(23,24)$. In addition, we investigated the indirect metastatic inhibitory effect by establishing a chemically induced adipocyte model from 3T3-L1 cell line differentiation which is a classical method avoiding lack of appropriate adipocytes $(25,26)$.

\section{Materials and methods}

Cell culture. The human lung fetal cell line MRC-5 was cultured in minimum essential media (MEM; Gibco). Mouse embryonic fibroblast 3T3-L1 cell line was cultured in Dulbecco's modified Eagle's medium (DMEM; Gibco). Human lung cancer cell line, 95D (with high metastatic potential) was cultured in RPMI-1640 medium (Gibco). All the cells were obtained from the Cell Bank of the Institute of Biochemistry and Cell Biology, Chinese Academy of Sciences (Shanghai, China). The media were supplemented with $10 \%$ fetal bovine serum (FBS; Gibco), $100 \mathrm{U} / \mathrm{ml}$ penicillin and $100 \mathrm{U} / \mathrm{ml}$ streptomycin. The cells were incubated in a humidified atmosphere with $95 \%$ air and $5 \% \mathrm{CO}_{2}$ at $37^{\circ} \mathrm{C}$.

Drugs and reagents. DT-13 powder was kindly provided by Professor Yu Boyang (China Pharmaceutical University). We purchased anti-paxilin, anti-phospho-paxiliin, anti-FAK, anti-phospho-FAK,anti-c-raf,anti-phospho-c-raf,anti-ERK,antiphospho-ERK, anti-HIF-1 $\alpha$, anti-NMIIA and FITC-conjugated secondary antibodies from Cell Signaling Technology. Anti$\alpha$-SMA was from Abcam. Methylisobutylxanthine (IBMX) and insulin (bovine) were purchased from Sigma. Dexamethasone was from G-Biosciences.

Conditioned medium (CM) preparation. The normoxic conditioned medium (Nor-CM) and hypoxic conditioned medium (Hypo-CM) were harvested from the condition of normoxic/ hypoxic MRC-5 cells or adipocytes, respectively. Then the conditioned medium was cleared through centrifugation. The $\mathrm{CM}$ was stored at $-80^{\circ} \mathrm{C}$ without repeated freezing and thawing.

To obtain Hypo-CM (DT-13) from DT-13-treated hypoxic MRC-5 cells or adipocytes, the MRC-5 cells or adipocytes were treated with DT-13 $(0.1,1$, and $10 \mu \mathrm{M})$ and cultured in normoxic condition with medium. Twelve hours later, the used medium was removed and the cells were washed twice with PBS. Subsequently, the cells were cultured with fresh medium for additional $12 \mathrm{~h}$ in a hypoxic condition. Then the Hypo-CM (DT-13) was harvested as described above.

\section{Migration assays}

Wound-healing assay. Cells were seeded onto 12-well plates and incubated in complete medium to $100 \%$ confluency for the experiment. Wounds were created in the plates using a pipette tip. Then the cells were washed twice with PBS, replaced with fresh serum-free media and treated with $\mathrm{CM}$ or/and reagent at specified concentrations for another $12 \mathrm{~h}$. Subsequently, cell migration following each treatment was observed via photomicrograph.

Transwell migration assay. Cell migration was determined in Transwell chambers $(8-\mu \mathrm{m}$ pore size; Corning, Corning, NY, USA). Briefly, the CM was added to 24-well plates (the lower chamber), while the cells were seeded into the upper chamber with serum-free medium. After $12 \mathrm{~h}$, the cells migrated through the membrane and adhered to the underside of the membrane. Subsequently, the migrated cells were stained with crystal violet and counted via photomicrograph at x400 magnification.

Invasion assay. Cancer cell invasion was assayed in Transwell chambers ( $8-\mu \mathrm{m}$ pore size; Corning). The membrane of the upper chamber was coated with $30 \mu \mathrm{g}$ of Matrigel (Sigma) for $2 \mathrm{~h}$. After $12 \mathrm{~h}$, the cells invaded through the Matrigel and membrane to the underside of the chamber membrane. Subsequently, the migrated cells were stained by crystal violet and counted via photomicrograph at $\mathrm{x} 400$ magnification.

Immunofluorescent staining. Cells were seeded on coverslips in 24-well plates and cultured to $50-60 \%$ confluency for the experiment. After $12 \mathrm{~h}$, the coverslips were washed with PBS, and then fixed in $4 \%$ paraformaldehyde for $20 \mathrm{~min}$ at room temperature. Next, the cells were washed and incubated with the antibody for $12 \mathrm{~h}$ at $4^{\circ} \mathrm{C}$. In a dark place, the cells were then incubated with a secondary antibody for $1 \mathrm{~h}$ at room temperature and with Hoechst 33342 for 30 min. Subsequently, the processed cells were mounted, and the results were analyzed via fluorescence photomicrograph by an inverted microscope (Olympus IX-71; Olympus, Japan).

Western blot analysis. Total cell lysates were extracted from the cultured cells with protease inhibitors. The proteins were fractionated by $8-12 \%$ sodium dodecyl sulfate-polyacrylamide gel electrophoresis (SDS-PAGE) and electroblotted onto PVDF membranes (Millipore, USA). Blocking buffer was used for blocking and the membranes were incubated with primary antibodies for $12 \mathrm{~h}$ at $4^{\circ} \mathrm{C}$. Then, it was probed with relative secondary antibody for $1 \mathrm{~h}$ at room temperature. Subsequently, the expression of the target immunoreactive proteins was examined by Immobilon Western Chemiluminescent HRP substrate (Millipore).

Statistical analysis. The results are expressed as the mean \pm standard deviation (SD). GraphPad Prism 5.0 and the Student's t-test were used to determine the level of significance. A p-value of $<0.05$ was considered to indicate a statistically significant result. 
A

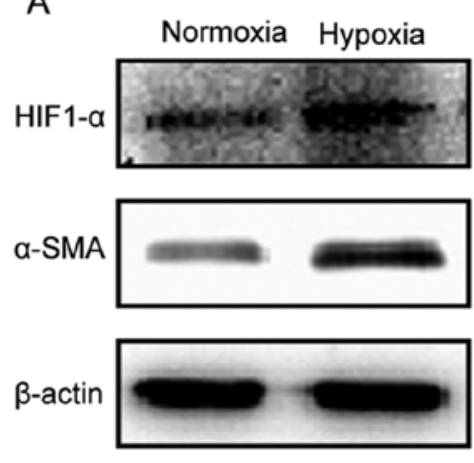

C

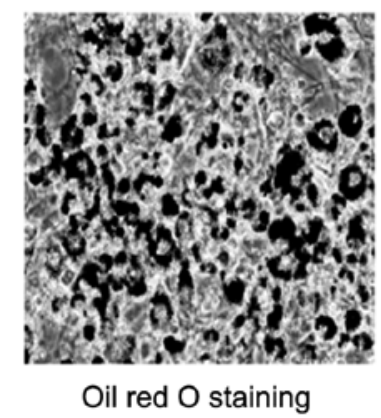

B

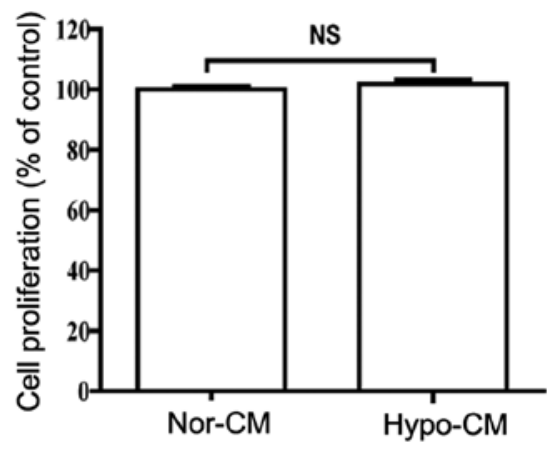

D

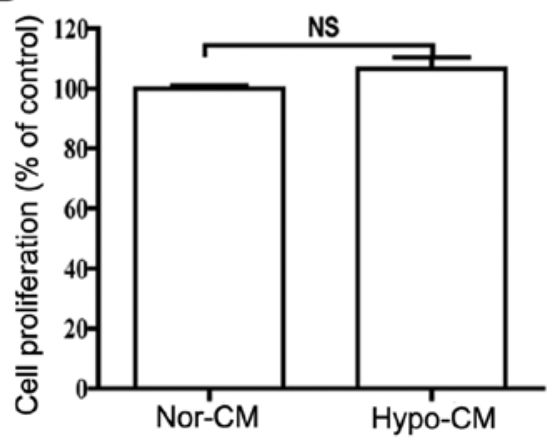

Figure 1. Establishment of the tumor microenvironment model through exposure to hypoxia or chemically induced differentiation. (A) The expression levels of $\alpha$-SMA and HIF-1 $\alpha$ protein in MRC-5 cells were upregulated by hypoxia. (B) Effects of conditioned medium (CM) from hypoxic MRC-5 cells on 95D cell proliferation were detected by MTT assay after the cancer cells were treated with Nor-CM and Hypo-CM for 12 h. (C) The chemically induced adipocytes were detected by Oil red O staining. The adipocytes were induced from 3T3-L1 cells by response to a mixture of insulin, dexamethasone, and IBMX (methylisobutylxanthine) during an 8-day procedure. (D) Effect of CM from hypoxic adipocytes on 95D cell proliferation was detected by MTT assay after the cancer cells were treated with Nor-CM and Hypo-CM for $12 \mathrm{~h}$. Data are expressed as mean \pm SD, compared with the Nor-CM. Nor-CM, normoxic conditioned medium; Hypo-CM, hypoxic conditioned medium.

\section{Results}

Establishment of the tumor microenvironment model through exposure to hypoxia and chemically induced differentiation. Activation of the $\alpha$-SMA gene is an essential feature during the conversion of fibroblasts into myofibroblasts. Therefore, we investigated the expression of $\alpha$-SMA protein in hypoxic MRC-5 cells, to characterize the shift of cultured fibroblasts. After hypoxia for $12 \mathrm{~h}$, the expression of $\alpha$-SMA was significantly upregulated, suggesting that hypoxic MRC-5 cells were analogous to CAFs (Fig. 1A) as previously reported $(23,24)$. In addition, HIF-1 $\alpha$ was confirmed to be the major transcriptional factor in response to hypoxia. Therefore, the upregulated level of HIF-1 $\alpha$ also demonstrated the response of MRC-5 cells to hypoxia (Fig. 1A). The cell proliferation assay showed that the CM from the hypoxic fibroblasts had no effect on cancer cell proliferation (Fig. 1B).

Meanwhile, the chemically induced adipocyte model was established according to published protocols by response to a mixture of insulin, dexamethasone and IBMX. After the 8-day procedure (25), the mature adipocytes were detected by Nile Red $\mathrm{O}$ staining (Fig. 1C), and then hypoxia for $12 \mathrm{~h}$. The cell proliferation assay showed that the CM from hypoxic adipocytes did not affect cancer cell proliferation (Fig. 1D). Obviously, the model which was established through exposure to hypoxia and chemically induced differentiation was analo- gous to CAFs and adipocytes, respectively, to some extent. In addition, $\mathrm{CM}$ is an appropriate mediator to explore the crosstalk between two cell types. Therefore, the model made it possible for us to evaluate the migration induced by the tumor microenvironment.

The conditioned medium from the tumor microenvironment model promotes migration and invasion. In the present study, wound-healing and Transwell chamber assays were used to examine the invasion and migration induced by Hypo-CM (hypoxic-conditioned medium). After 95D cells were treated with Hypo-CM from the fibroblasts for $12 \mathrm{~h}$, the migration distance increased compared with the Norm-CM group (Fig. 2A). Meanwhile, a higher percentage of cancer cells moved across the membrane to the underside when compared with that noted in the Norm-CM group (Fig. 2A), after treatment with Hypo-CM for 12 h. Similarly, Hypo-CM from adipocytes significantly promoted the migration distance and the migration number of 95D cells compared with the Norm-CM group, after treatment with Hypo-CM from adipocytes for $12 \mathrm{~h}$ (Fig. 2B). However, Hypo-CM from the fibroblasts also stimulated the invasion of the 95D cells (Fig. 2A).

In addition, we investigated the distribution of NMIIA in cancer cells affected by Hypo-CM from hypoxic fibroblasts and adipocytes via immunofluorescent staining, to confirm whether cell migration ability could be improved via regulation 


\section{A}
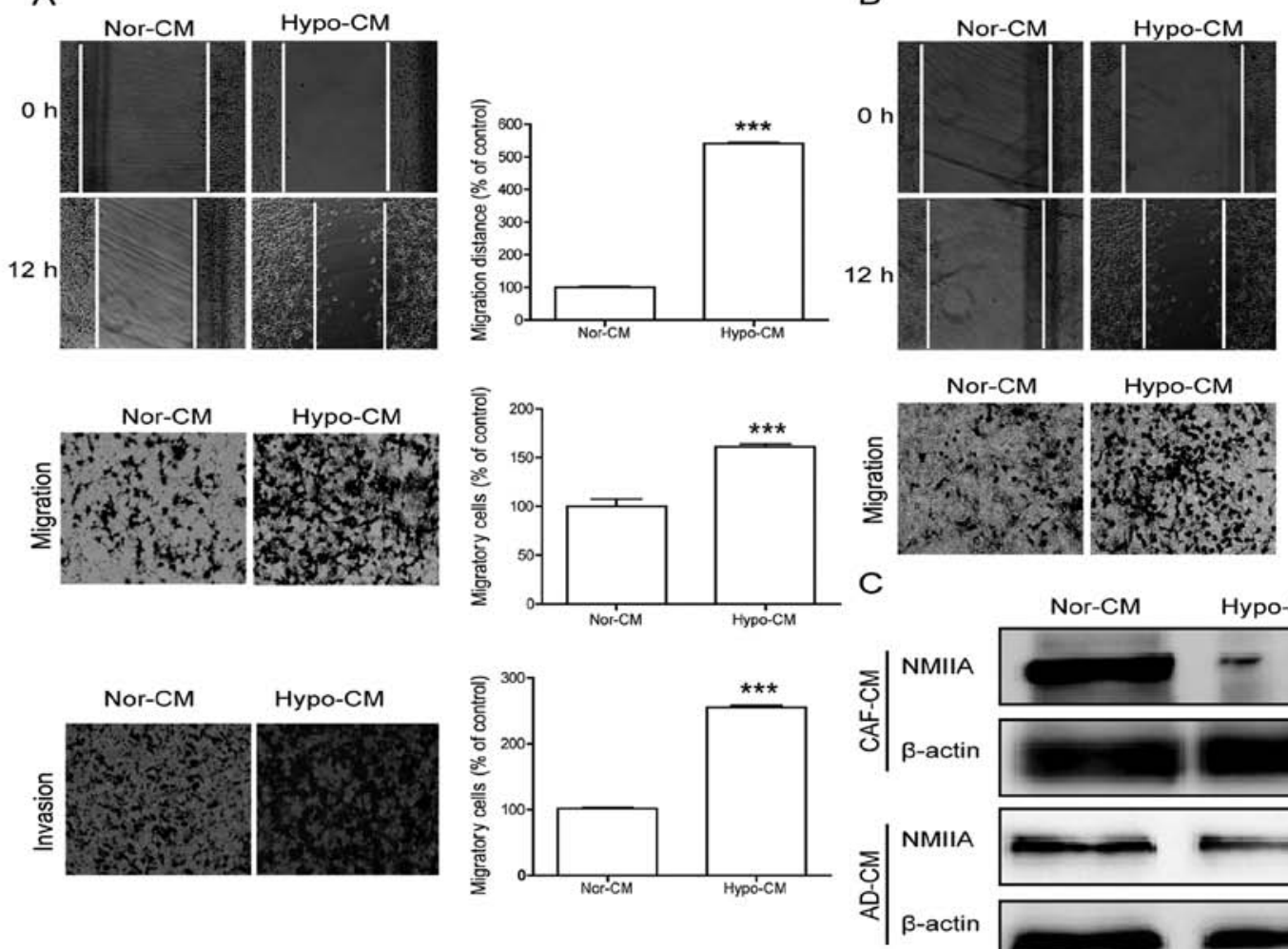

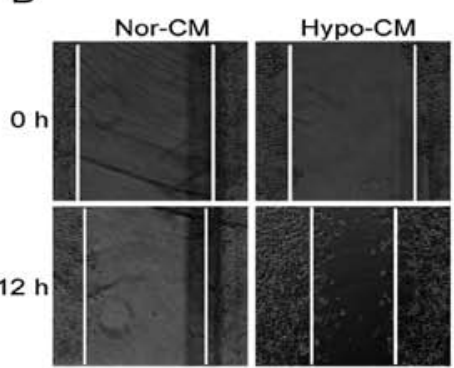

Nor-CM

B
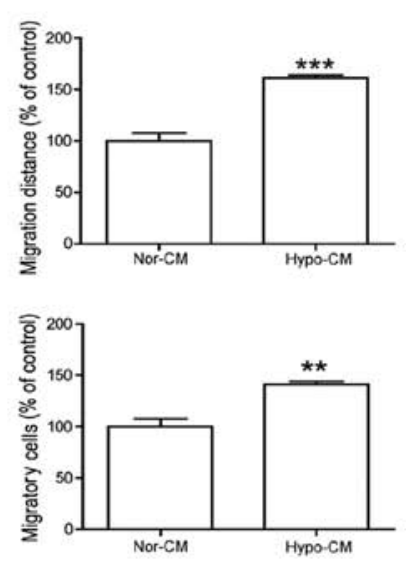

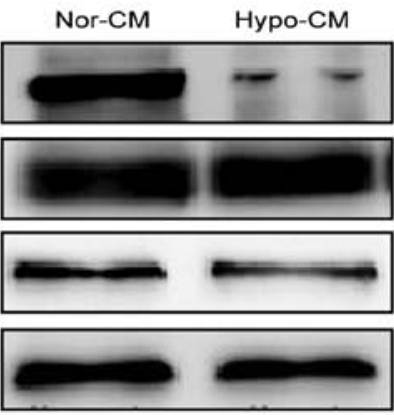

Figure 2. Conditioned medium (CM) from the tumor microenvironment model promotes the migration and invasion of cancer cells. (A) Hypo-CM from fibroblasts promoted the migration and invasion of 95D cells. The effects were detected by wound-healing, Transwell migration and Transwell invasion assays, respectively. (B) Hypo-CM from adipocytes promoted the the migration of 95D cells. The effects were detected by wound-healing and Transwell migration assays, respectively. (C) Hypo-CM from hypoxic fibroblasts and adipocytes inhibited NMIIA in 95D cells as determined by western blot analysis. The cancer cells were treated with Nor-CM and Hypo-CM for $12 \mathrm{~h}$. Data are expressed as mean $\pm \mathrm{SD},{ }^{* * * *} \mathrm{P}<0.001,{ }^{* *} \mathrm{P}<0.01$, compared with the Nor-CM. Nor-CM, normoxic conditioned medium; Hypo-CM, hypoxic conditioned medium.

of NMIIA in the cancer cells. Ultimately, the photomicrograph showed that NMIIA spread to the cytoplasm after treatment with Hypo-CM from the tumor microenvironment model, while NMIIA closely accumulated around the nucleus in the control (Fig. 4C). Further western blot analysis showed that Hypo-CM from the tumor microenvironment model significantly inhibited NMIIA, confirming that low expression of NMIIA can promote cancer cell motility and migration as in previous research (15-17) (Fig. 2C). Obviously, all these results indicated that hypoxic fibroblasts and adipocytes were able to promote 95D migration by regulating NMIIA.

NMIIA regulates the migration and invasion of cancer cells in the tumor microenvironment model. Downregulation of NMIIA can promote cancer cell motility and migration via lamellipodia extension, decrease in focal adhesions, and upregulation of paxillin/p-paxillin. In addition, blebbistatin, an inhibitor of non-muscle myosin II, can promote cell migration by inhibiting NMIIA but has no effect on the proliferation of cancer cells (27). After concomitant treatment with Hypo-CM from hypoxic fibroblasts and blebbistatin for $12 \mathrm{~h}$, the cell migration was improved, compared with that treated only with Hypo-CM or Norm-CM via wound-healing and Transwell chamber assays (Fig. 3A). However, the cell invasion was slightly reduced compared with the Hypo-CM group, but significantly improved in comparison with the Norm-CM group (Fig. 3A). Meanwhile, the Transwell migration assay also confirmed that the inhibition of NMIIA could promote 95D cell migration in the condition of hypoxic adipocytes (Fig. 3B). In brief, the above results suggested that NMIIA plays a role in cell motility and migration in the hypoxic tumor microenvironment; thus, indicating that NMIIA is a significant potential target for anti-metastasis.

DT-13 inhibits the migration and invasion of cancer cells indirectly in the tumor microenvironment model. DT-13 has been found to exhibit an anti-metastatic effect in vitro and in vivo. As a natural traditional Chinese medicine, it inhibits cell migration via multi-levels, different pathways and multiple targets. Our previous research showed that DT-13 could exhibit antitumor effects directly by regulating NMIIA in cancer cells (22). Firstly, the cell proliferation assay showed that DT-13 had no effect on the proliferation of MRC-5 cells (data not shown). However, the Hypo-CM (DT-13) from hypoxic fibroblasts reduced cell migration and invasion significantly (Fig. 4A), but without a proliferation effect on the cancer cells (data not shown). Meanwhile, the Transwell migration assay also confirmed that DT-13 could inhibit 95D cell migration in the condition of hypoxic adipocytes (Fig. 4B). Furthermore, the immunofluorescent staining showed that DT-13 could reverse the Hypo-CM-induced spread of NMIIA to the cytoplasm both in the condition of hypoxic fibroblasts and adipocytes (Fig. 4C). 

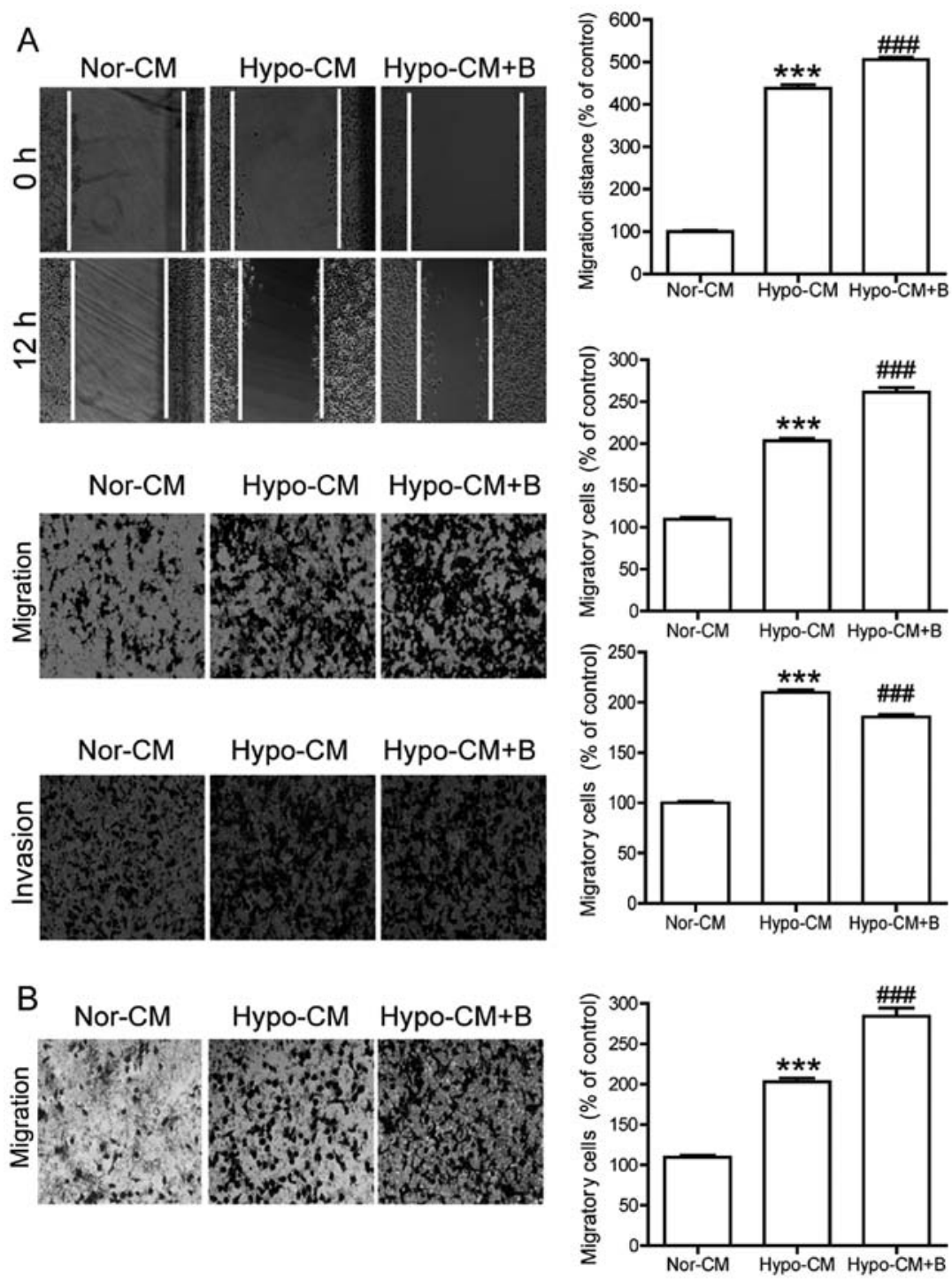

Figure 3. NMIIA regulates the migration and invasion of cancer cells. (A) Blebbistatin further promoted cancer cell migration and invasion as detected by wound-healing, Transwell migration and Transwell invasion assays, respectively, indirectly in the hypoxic fibroblast microenvironment. (B) Blebbistatin further promoted cancer cell migration as detected by Transwell migration assay indirectly in the hypoxic adipocyte microenvironment. The cancer cells were treated with Nor-CM, Hypo-CM or/and blebbistatin $(+\mathrm{B})$, respectively, for $12 \mathrm{~h}$. Data are expressed as mean $\pm \mathrm{SD},{ }^{* * *} \mathrm{P}<0.001,{ }^{* *} \mathrm{P}<0.01$, compared with the Nor-CM; ${ }^{\# \#} \mathrm{P}<0.001,{ }^{\# \#} \mathrm{P}<0.01$, compared with the Hypo-CM. Nor-CM, normoxic conditioned medium; Hypo-CM, hypoxic conditioned medium.

Therefore, all these results indicated that DT-13 inhibits cell migration and invasion by regulating NMIIA in cancer cells indirectly in the tumor microenvironment model.

DT-13 inhibits cell migration by regulating NMIIA in cancer cells indirectly in the tumor microenvironment model. NMIIA can regulate the migration and invasion of cancer cells in the hypoxic tumor microenvironment. Meanwhile, DT-13 inhibits metastasis indirectly by hypoxic fibroblasts and adipocytes. Therefore, western blot analysis was used to investigate whether NMIIA and downstream proteins could be affected by Hypo-CM (DT-13). Notably, Hypo-CM (DT-13) upregulated NMIIA, while its expression was inhibited by Hypo-CM. p-FAK was inhibited by Hypo-CM, but was significantly upregulated by Hypo-CM (DT-13) compared with the Hypo-CM group, under conditions of hypoxic fibroblasts and adipocytes (Fig. 5A and C). Paxillin and p-paxillin had no change in the hypoxic fibroblast microenvironment, while
Hypo-CM (DT-13) significantly inhibited p-paxillin induced by Hypo-CM from the adipocytes (Fig. 5A and C). Obviously, these data suggest that DT-13 inhibits cell migration by regulating NMIIA in cancer cells indirectly in a hypoxic tumor microenvironment.

Next, we ascertained whether the regulatory pathway of NMIIA in cancer cells was affected by Hypo-CM (DT-13). Western blot analysis found that p-c-Raf and p-ERK1/2 were upregulated by Hypo-CM, while the phosphorylation levels of both were greatly inhibited by Hypo-CM (DT-13) (Fig. 5B and D), indicating that DT-13 regulates NMIIA via the Raf-ERK1/2 signaling pathway in cancer cells after Hypo-CM (DT-13) treatment.

\section{Discussion}

The tumor microenvironment has been increasingly confirmed as a key factor in multiple stages of cancer progres- 

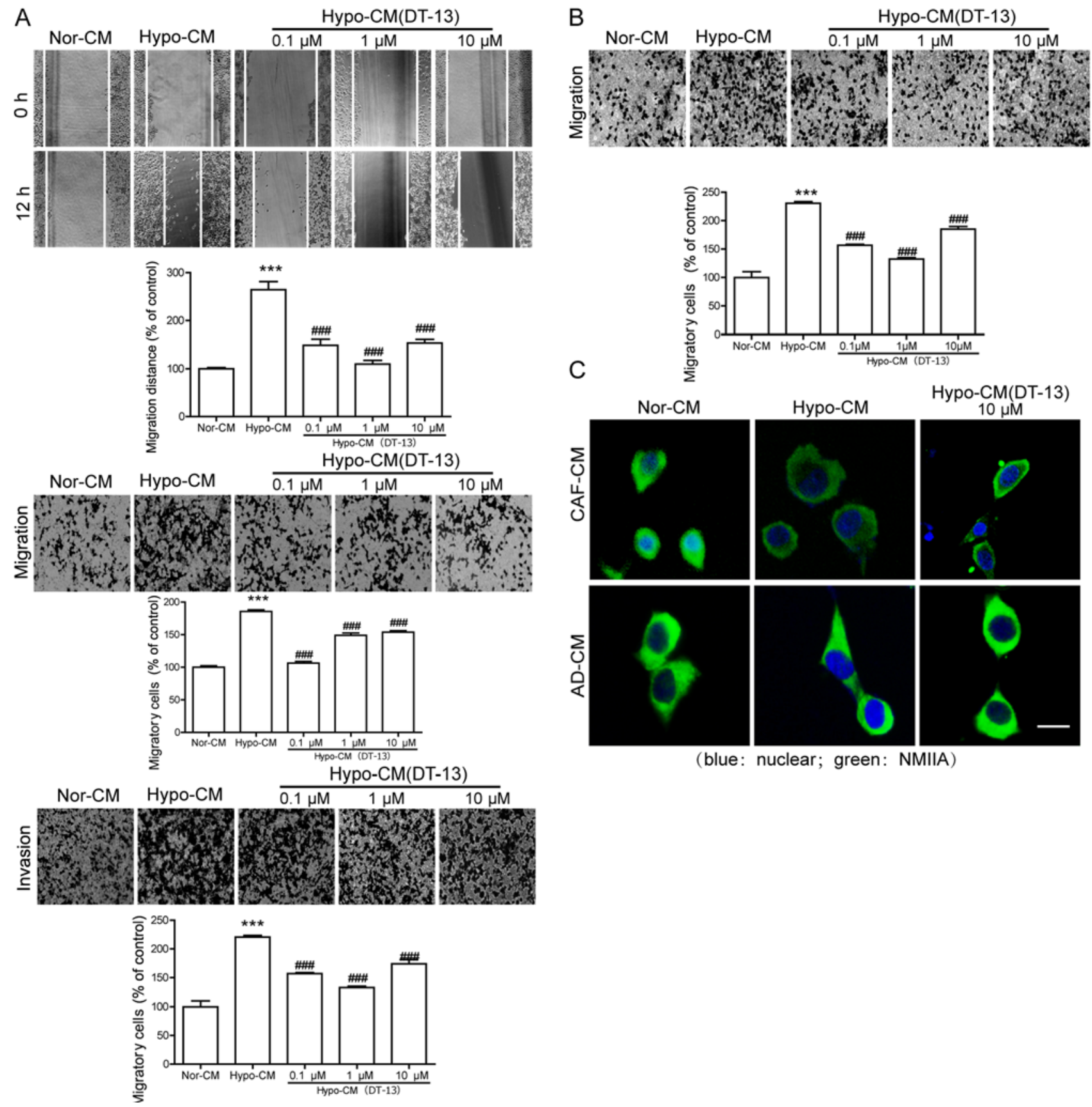

Figure 4. DT-13 inhibits the migration and invasion of cancer cells indirectly in the tumor microenvironment model. (A) Hypo-CM (DT-13) (CM from DT-13treated hypoxic fibroblasts) inhibited the migration and invasion of cancer cells as detected by wound-healing, Transwell migration and Transwell invasion assays, respectively, indirectly in hypoxic fibroblast microenvironment. (B) Hypo-CM (DT-13) (CM from DT-13-treated hypoxic adipocytes) inhibited the migration of cancer cells as detected by Transwell migration assay. (C) Hypo-CM (DT-13) (CM from DT-13-treated hypoxic fibroblasts or adipocytes) inhibited the spread of NMIIA toward the cytoplasm by IF analysis. The cancer cells were treated with Nor-CM, Hypo-CM or Hypo-CM (DT-13), respectively, for $12 \mathrm{~h}$. Data are expressed as mean $\pm \mathrm{SD},{ }^{* * * *} \mathrm{P}<0.001,{ }^{* *} \mathrm{P}<0.01$, compared with the Nor-CM; ${ }^{\# \# *} \mathrm{P}<0.001,{ }^{\# \#} \mathrm{P}<0.01$, compared with the Hypo-CM.

sion (2). Thereby, more therapeutic strategies are purposefully designed to not only target cancer cells but also regulate the tumor microenvironment (5). Notably, DT-13 has been found to exhibit antitumor effects, especially anti-metastasis in vitro and in vivo. Moreover, our research showed that DT-13 can exhibit anti-metastasis effects directly by regulating NMIIA especially in lung cancer cells 95D (22). Therefore, it is not known whether DT-13 can also inhibit cell migration by regulating NMIIA in cancer cells indirectly in the tumor microenvironment.
Fibroblasts and adipocytes are two most representative mesenchymal cells in the tumor microenvironment (4). We established a CAF-like myofibroblastic phenotype model and a chemically induced adipocyte model as previously reported (23-25), revealing the effects of the tumor microenvironment on cancer cells to some extent. In this model, the conditioned medium from the tumor microenvironment model significantly promoted $95 \mathrm{D}$ cell migration and regulated the expression of NMIIA. Then, we confirmed the hypothesis that the tumor microenvironment regulates NMIIA in cancer cells 
A

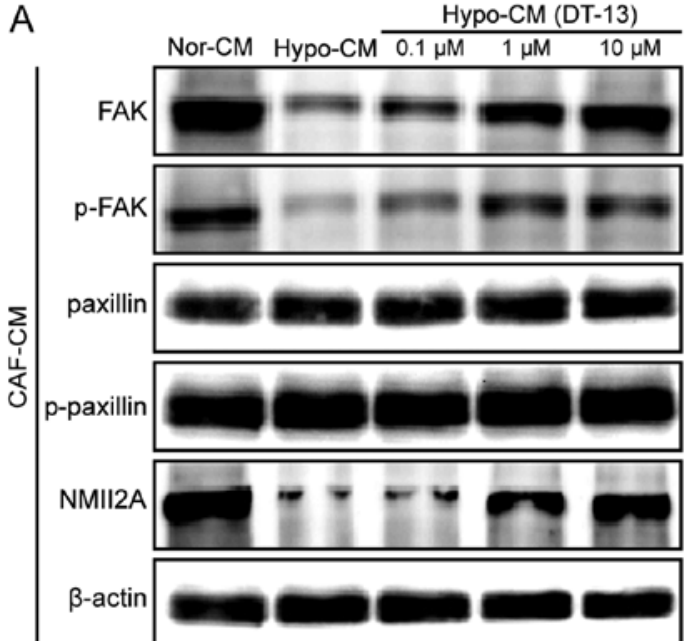

B

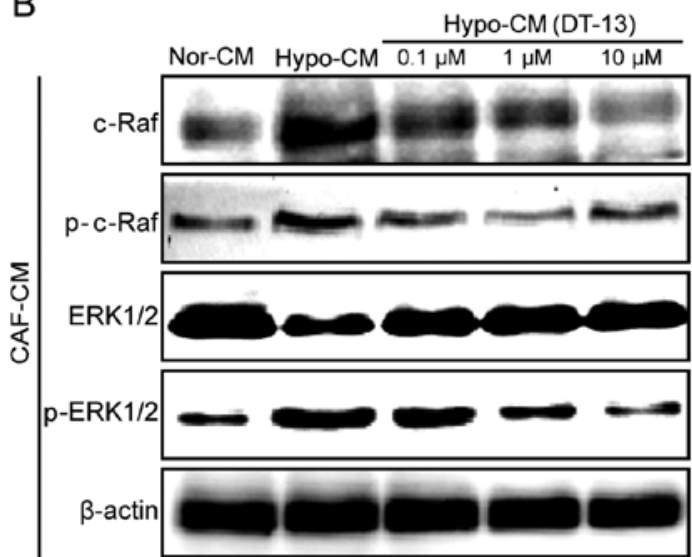

C

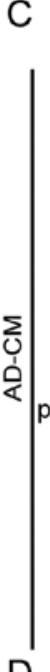



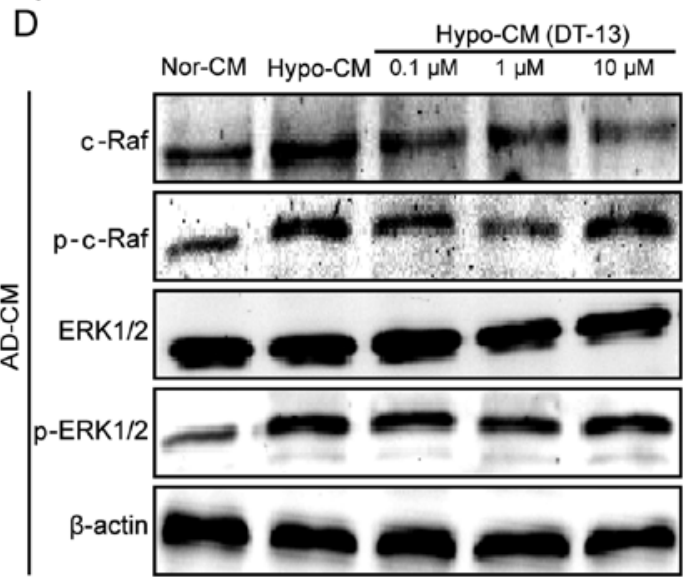

Figure 5. DT-13 inhibits migration via the regulation of NMIIA through the c-raf/ERK pathways in cancer cells indirectly in the tumor microenvironment model. (A) The expression of NMIIA and its downstream proteins was determined under conditions of hypoxic fibroblasts. (B) The expression of NMIIA and its downstream proteins was determined under conditions of hypoxic adipocytes. (C) The expression of c-raf/ERK pathway proteins was determined under conditions of hypoxic fibroblasts. (D) The expression of c-raf/ERK pathway proteins was determined under conditions of hypoxic adipocytes. The cancer cells were treated with Nor-CM, Hypo-CM or Hypo-CM (DT-13) under conditions of hypoxic fibroblasts (CAF-CM; A and B) or adipocytes (AD-CM; C and D), respectively, for $12 \mathrm{~h}$, and then the proteins were analyzed by western blot analysis.

and facilitates migration by using the non-muscle myosin II inhibitor, blebbistatin, in further experiments. Importantly, this is the first report to the best of our knowledge that the tumor microenvironment can promote cancer cell migration by regulating the expression of NMIIA. Finally, DT-13 was found to inhibit cell migration by regulating NMIIA and its downstream proteins in cancer cells indirectly in the tumor microenvironment model. Further results showed that DT-13 exhibited anti-migratory effects via the raf/ERK1/2 signal pathway. Consequently, the study confirmed that DT-13 significantly inhibited 95D cell migration in vitro, showing potential anti-metastatic effects on lung cancer and the scientific basis for drug development.

Mesenchymal cells include CAFs, adipocytes, immune cells, inflammatory cells and endothelial cells. T cells, B cells, macrophages and mast cells are immune cells which can also promote immune escape, tumor growth and metastasis (28). Inflammatory cells can secrete excessive amounts of inflammatory cytokines such as IL-6, IL-10, TGF- $\beta$, EGF to stimulate invasion, migration, proliferation, to enhance other mesenchymal cells inducing metastasis (29). Under the condition of tumor angiogenesis factors, endothelial cells can establish tumor vasculature to support cancer metastasis and proliferation, aggravating tumor malignancy (30). In brief, immune cells, inflammatory cells, endothelial cells and other mesenchymal cells compose the complex tumor microenvironment with CAFs and adipocytes. Together they promote cancer cell migration and invasion.

NMIIA is a significant potential target for anti-metastasis in highly metastatic cancer (31). DT-13 has been found to exhibit an anti-metastatic effect directly by regulating NMIIA in 95D cells. In the present study, the data suggested that DT-13 can also inhibit 95D cell migration by regulating the mechanism of cancer cells indirectly by hypoxic fibroblasts and adipocytes. Whether DT-13 can inhibit cancer cell migration indirectly through immune cells, inflammatory cells or endothelial cells warrants further investigation. The filament assembly of NMIIA is selectively regulated by certain S100 proteins such as S100A4 (the small $\mathrm{Ca}^{2+}$-binding protein) which can enhance cell migration in certain types of cancer $(32,33)$. Thus, further research will determine the effect of DT-13 on S100A4. Moreover, subsequent experiments should confirm the in vitro mechanism by establishment of orthotopic tumor models and observation by PET/CT (34). 
Although metastases are responsible for the majority of cancer-related deaths, there are few therapeutic approaches that specifically target metastasis. On the basis of these findings, the tumor microenvironment is further confirmed as a novel strategy by which to inhibit tumor metastases. Importantly, DT-13 may be a potential anti-metastatic drug for clinical application.

\section{Acknowledgements}

This study was funded by the National Natural Science Foundation of China for Youth (no. 81102853), the National Natural Science Foundation of China (no. 81573456) and the National High-Tech Research and Development Projects (863) (no. 2014AA022208).

\section{References}

1. Chen W, Zheng R, Zhang S, Zhao P, Zeng H, Zou X and He J: Annual report on status of cancer in China, 2010. Chin J Cancer Res 26: 48-58, 2014

2. Hanahan D and Weinberg RA: Hallmarks of cancer: The next generation. Cell 144: 646-674, 2011.

3. Leonardi GC, Candido S, Cervello M, Nicolosi D, Raiti F, Travali S, Spandidos DA and Libra M: The tumor microenvironment in hepatocellular carcinoma (Review). Int J Oncol 40 1733-1747, 2012.

4. Semenza GL: The hypoxic tumor microenvironment: A driving force for breast cancer progression. Biochim Biophys Acta 1863 : 382-391, 2016.

5. De Wever O, Van Bockstal M, Mareel M, Hendrix A and Bracke M: Carcinoma-associated fibroblasts provide operational flexibility in metastasis. Semin Cancer Biol 25: 33-46, 2014.

6. Routray S, Sunkavali A and Bari KA: Carcinoma-associated fibroblasts, its implication in head and neck squamous cell carcinoma: A mini review. Oral Dis 20: 246-253, 2014.

7. Ali AT, Hochfeld WE, Myburgh R and Pepper MS: Adipocyte and adipogenesis. Eur J Cell Biol 92: 229-236, 2013.

8. Bochet L, Meulle A, Imbert S, Salles B, Valet P and Muller C: Cancer-associated adipocytes promotes breast tumor radioresistance. Biochem Biophys Res Commun 411: 102-106, 2011.

9. Chiu HC, Chang TY, Huang CT, Chao YS and Hsu JT: EGFR and myosin II inhibitors cooperate to suppress EGFR-T790M-mutant NSCLC cells. Mol Oncol 6: 299-310, 2012.

10. Gupton SL and Waterman-Storer CM: Spatiotemporal feedback between actomyosin and focal-adhesion systems optimizes rapid cell migration. Cell 125: 1361-1374, 2006.

11. Even-Ram S, Doyle AD, Conti MA, Matsumoto K, Adelstein RS and Yamada KM: Myosin IIA regulates cell motility and actomyosin-microtubule crosstalk. Nat Cell Biol 9: 299-309, 2007.

12. Hosono Y, Usukura J, Yamaguchi T, Yanagisawa K, Suzuki M and Takahashi T: MYBPH inhibits NM IIA assembly via direct interaction with NMHC IIA and reduces cell motility. Biochem Biophys Res Commun 428: 173-178, 2012.

13. Kim JH and Adelstein RS: LPA(1)-induced migration requires nonmuscle myosin II light chain phosphorylation in breast cancer cells. J Cell Physiol 226: 2881-2893, 2011.

14. Vicente-Manzanares M, Ma X, Adelstein RS and Horwitz AR: Non-muscle myosin II takes centre stage in cell adhesion and migration. Nat Rev Mol Cell Biol 10: 778-790, 2009.

15. Jacobelli J, Friedman RS, Conti MA, Lennon-Dumenil AM, Piel M, Sorensen CM, Adelstein RS and Krummel MF: Confinement-optimized three-dimensional $\mathrm{T}$ cell amoeboid motility is modulated via myosin IIA-regulated adhesions. Nat Immunol 11: 953-961, 2010.

16. Tanaka C, Ito S, Nishio N, Kodera Y, Sakurai H, Suzuki H, Nakao A and Isobe K: GADD34 suppresses wound healing by upregulating expression of myosin IIA. Transgenic Res 19: 637-645, 2010

17. Betapudi V: Myosin II motor proteins with different functions determine the fate of lamellipodia extension during cell spreading. PLoS One 5: e8560, 2010.
18. Zhang Y, Liu J, Kou J, Yu J and Yu B: DT-13 suppresses MDA-MB-435 cell adhesion and invasion by inhibiting MMP-2/9 via the p38 MAPK pathway. Mol Med Rep 6: 1121-1125, 2012.

19. Zhao R, Sun L, Lin S, Bai X, Yu B, Yuan S and Zhang L: The saponin monomer of dwarf lilyturf tuber, DT-13, inhibits angiogenesis under hypoxia and normoxia via multi-targeting activity. Oncol Rep 29: 1379-1386, 2013.

20. Ren-Ping Z, Sen-Sen L, Yuan ST, Yu BY, Bai XS, Sun L and Zhang LY: DT-13, a saponin of dwarf lilyturf tuber, exhibits anti-cancer activity by down-regulating $\mathrm{C}-\mathrm{C}$ chemokine receptor type 5 and vascular endothelial growth factor in MDA-MB-435 cells. Chin J Nat Med 12: 24-29, 2014.

21. Lin SS, Fan W, Sun L, Li FF, Zhao RP, Zhang LY, Yu BY and Yuan ST: The saponin DT-13 inhibits gastric cancer cell migration through down-regulation of CCR5-CCL5 axis. Chin J Nat Med 12: 833-840, 2014.

22. Wei X, Lin S, Liu Y, Zhao R, Ghulam JK, Du H, Mao T, Yu B, Yuan S and Sun L: DT-13 attenuates lung cancer metastasis via directly regulating NMIIA activity under hypoxia condition. Oncol Rep (In press).

23. Fang D, Sun L, Lin S, Zhou L, Su N, Yuan S and Yu B: Vinorelbine inhibits angiogenesis and 95D migration via reducing hypoxic fibroblast stromal cell-derived factor 1 secretion. Exp Biol Med (Maywood) 237: 1045-1055, 2012.

24. Zhou L, Sun L, Lin S, Fang D, Żhao R, Zhu J, Liu J, Chen L, Shi W, Yuan S, et al: Inhibition of angiogenic activity of hypoxic fibroblast cell line MRC-5 in vitro by topotecan. Med Oncol: Nov 9, 2010 (Epub ahead of print).

25. Choi KC, Lee SY, Yoo HJ, Ryu OH, Lee KW, Kim SM, Baik SH and Choi KM: Effect of PPAR-delta agonist on the expression of visfatin, adiponectin, and resistin in rat adipose tissue and 3T3-L1 adipocytes. Biochem Biophys Res Commun 357: 62-67, 2007.

26. Gernapudi R, Yao Y, Zhang Y, Wolfson B, Roy S, Duru N, Eades G, Yang $P$ and Zhou Q: Targeting exosomes from preadipocytes inhibits preadipocyte to cancer stem cell signaling in early-stage breast cancer. Breast Cancer Res Treat 150: 685-695, 2015.

27. Jean L, Majumdar D, Shi M, Hinkle LE, Diggins NL, Ao M, Broussard JA, Evans JC, Choma DP and Webb DJ: Activation of Rac by Asef 2 promotes myosin II-dependent contractility to inhibit cell migration on type I collagen. J Cell Sci 126: 5585-5597, 2013.

28. Semov A, Moreno MJ, Onichtchenko A, Abulrob A, Ball M, Ekiel I, Pietrzynski G, Stanimirovic D and Alakhov V: Metastasis-associated protein S100A4 induces angiogenesis through interaction with Annexin II and accelerated plasmin formation. J Biol Chem 280: 20833-20841, 2005.

29. Sun Z, Wang S and Zhao RC: The roles of mesenchymal stem cells in tumor inflammatory microenvironment. J Hematol Oncol 7: 14, 2014.

30. Du H, Shi H, Chen D, Zhou Y and Che G: Cross-talk between endothelial and tumor cells via basic fibroblast growth factor and vascular endothelial growth factor signaling promotes lung cancer growth and angiogenesis. Oncol Lett 9: 1089-1094, 2015.

31. Derycke L, Stove C, Vercoutter-Edouart AS, De Wever O, Dollé L, Colpaert N, Depypere H, Michalski JC and Bracke M: The role of non-muscle myosin IIA in aggregation and invasion of human MCF-7 breast cancer cells. Int J Dev Biol 55: 835-840, 2011.

32. Elliott PR, Irvine AF, Jung HS, Tozawa K, Pastok MW, Picone R, Badyal SK, Basran J, Rudland PS, Barraclough R, et al: Asymmetric mode of $\mathrm{Ca}^{2+}{ }_{-} \mathrm{S} 100 \mathrm{~A} 4$ interaction with nonmuscle myosin IIA generates nanomolar affinity required for filament remodeling. Structure 20: 654-666, 2012.

33. Bowers RR, Manevich Y, Townsend DM and Tew KD: Sulfiredoxin redox-sensitive interaction with S100A4 and non-muscle myosin IIA regulates cancer cell motility. Biochemistry 51: 7740-7754, 2012.

34. Takahashi O, Komaki R, Smith PD, Jürgensmeier JM, Ryan A, Bekele BN, Wistuba II, Jacoby JJ, Korshunova MV, Biernacka A, et al: Combined MEK and VEGFR inhibition in orthotopic human lung cancer models results in enhanced inhibition of tumor angiogenesis, growth, and metastasis. Clin Cancer Res 18: 1641-1654, 2012. 